\section{Effect of oral azithromycin in the treatment of chlamydial conjunctivitis}

\begin{abstract}
Purpose To assess the efficacy of oral azithromycin in the treatment of chlamydial conjunctivitis.

Methods We performed a retrospective study in patients with clinically suspected chlamydial conjunctivitis who underwent conjunctival swab sampling for Chlamydia direct fluorescent antibody (DFA) tests between 1 January 2006 and 31 December 2006. Patients with positive DFA results were orally administered azithromycin once a week for 2 consecutive weeks. If DFA examinations still showed positive results after 4 weeks, additional azithromycin was orally administered once. The DFA tests were repeated 4 weeks later, and this was continued until the DFA tests showed negative results.
\end{abstract}

Results Among the 67 suspected patients, 45 $(67.2 \%)$ showed positive results from the DFA tests, of whom 42 received treatment. After the first 2 weeks, only 27 patients returned to the clinic and completed the treatment. The test results of $19(70.4 \%)$ patients became negative after the treatment with two weekly doses of oral azithromycin. Among the remaining eight patients, four (14.8\%) needed an additional dose of oral azithromycin, and the other four (14.8\%) required two additional doses. All 27 patients tolerated the treatment well, with an adverse event of mild gastritis in only one patient. Conclusions Two weekly doses of oral azithromycin were effective and well tolerated in the treatment of chlamydial conjunctivitis. However, more than one course of treatment was necessary in some patients.

Eye (2010) 24, 985-989; doi:10.1038/eye.2009.264; published online 6 November 2009

Keywords: azithromycin; chlamydial conjunctivitis; chlamydia trachomatis
Y-M Chen ${ }^{1,2}, \mathrm{~F}-\mathrm{R} \mathrm{Hu}{ }^{1}$ and $\mathrm{Y}-\mathrm{C} \mathrm{Hou}^{1}$

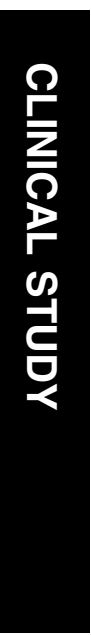

Gram-negative eubacterium that can cause a wide variety of diseases and constitutes a considerable public health problem.

C. trachomatis serotypes A, B, Ba, and C cause trachoma, which is endemic in many countries and also the leading infectious cause of blindness in the developing world.

C. trachomatis serotypes D-K cause adult or neonatal inclusion conjunctivitis, and are among of the major causes of sexually transmitted diseases in the developed countries. ${ }^{1,2}$ Inclusion conjunctivitis from C. trachomatis infection can present as redness of the eye with mucopurulent discharge, marked hyperemia, papillary hypertrophy, and a predominant follicular conjunctivitis. ${ }^{3,4}$ In the 1950s, tetracycline and erythromycin were found to be effective against $C$. trachomatis and replaced the less satisfactory sulpha drugs in the treatment of trachoma. ${ }^{5}$ Since then, topical tetracycline ointments have been widely used in many countries to control trachoma. The recommended treatment of trachoma is topical tetracycline ointment twice daily for 4-6 weeks ${ }^{1}$ or oral tetracycline/doxycycline/ erythromycin for few weeks. However, tetracycline ointment is irritating and difficult to use, and therefore, the compliance may be poor. ${ }^{1}$ The use of oral tetracycline, doxycycline, or erythromycin treatment requires a course of at least 7 days to 4 weeks and, thus, the compliance may also be poor. Since the early 1990s, several studies have been reported that single doses of oral azithromycin are effective in the treatment of trachoma. ${ }^{6-9}$ The World Health Organization now also advocates the use of oral azithromycin in the 'SAFE' strategy as an antibiotic for the control of trachoma worldwide. ${ }^{10}$ For C. trachomatis-induced inclusion conjunctivitis, ${ }^{11}$ because of its high
'Department of Ophthalmology, National Taiwan University Hospital, College of Medicine, National Taiwan University, Taipei, Taiwan

${ }^{2}$ Department of Ophthalmology, E-Da Hospital, I- Shou University, Kaohsiung, Taiwan

Correspondence: Y-C Hou, Department of Ophthalmology, National Taiwan University Hospital,

College of Medicine, National Taiwan University, No. 7, Chung-Shan South Road,

Taipei, Taiwan 100,

Taiwan

Tel: + 886223123456 ext. 5205;

Fax: + 886223934420

E-mail: ychou51@

ntu.edu.tw

Received: 12 December 2008

Accepted in revised form: 30 September 2009

Published online:

6 November 2009 
prevalence and association with genital tract infection, systemic treatment with oral antibiotics is preferred. ${ }^{12}$ Because azithromycin, an antibiotic, is intracellularly active and exhibits excellent activity against $C$. trachomatis in vitro, ${ }^{13}$ it is now also used for the treatment of C. trachomatis-induced inclusion conjunctivitis and even neonatal conjunctivitis. ${ }^{11,14}$ The efficacy of oral azithromycin has already been shown in the treatment of both trachoma ${ }^{6-9}$ and adult inclusion conjunctivitis ${ }^{11,15}$ in many studies. Although Taiwan was once an endemic area for trachoma in the 1950s and 1960s, ${ }^{16,17}$ the prevalence of trachoma in children had decreased to $15 \%$ in $1995 .{ }^{18}$ Factors such as the use of antibiotic ointments, education in personal hygiene, and improvements in the environment might have contributed to the decrease in the disease prevalence in Taiwan, but some sporadic cases of chlamydial conjunctivitis are still reported. Therefore, we have evaluated the efficacy of oral azithromycin in the treatment of patients with chlamydial conjunctivitis in the setting of our clinic.

\section{Materials and methods}

We performed a retrospective and non-randomized study to evaluate the efficacy of oral azithromycin in the treatment of chlamydial conjunctivitis. We reviewed the medical records of all patients with clinically suspected chlamydial conjunctivitis at the outpatient clinic of Dr Hou at National Taiwan University Hospital (NTUH) between 1 January 2006 and 31 December 2006. If patients had symptoms of eye redness, discharge, and irritation with the presentation of follicular conjunctivitis, conjunctival scarring, or corneal pannus formation, a diagnosis for chlamydial conjunctivitis was considered. We performed direct fluorescent antibody (DFA) tests for Chlamydia by swabbing across the lower and upper tarsal conjunctiva four times after topical application of $0.5 \%$ proparacaine. Chlamydia DFA reagent (bioMérieux, Marcy I'Etoile, France) was used for the DFA tests in the central laboratory of our hospital. All of the DFA tests were examined by the same experienced microbiologist who was masked to the identities and clinical conditions of the patients. Each DFA slide was read under a fluorescent microscope and was observed for discrete fluorescent chlamydial elementary bodies (EBs).

Table 1 Baseline patient data

\begin{tabular}{lcc}
\hline Characteristics & With azithromycin treatment & Evaluated \\
\hline No. of patients & 42 & 27 \\
Gender (male/female) & $20 / 22$ & $13 / 14$ \\
Age (years) & $36 \pm 22$ & $40 \pm 22$ \\
\hline
\end{tabular}

${ }^{\mathrm{a}}$ Mean \pm standard deviation.
The DFA test was considered positive if $\geqslant 10$ EBs were counted per high-power field. All patients with positive DFA results were orally administered azithromycin, except those who were pregnant, lactating, or had a history of allergy to macrolides. The patients were orally administered azithromycin ( $1000 \mathrm{mg}$ or $20 \mathrm{mg} / \mathrm{kg}$ ) once a week for 2 consecutive weeks, and the DFA tests were repeated 4 weeks after the treatment. If the DFA tests still showed positive results, an additional dose of azithromycin was orally administered, and another DFA test was performed again 4 weeks later. The augmented treatment with oral azithromycin (administration of one oral dose followed by DFA testing 4 weeks later) was continued until the DFA tests showed negative results. The adverse events recorded in the medical charts were reviewed as well. The study was approved by the institutional review board of NTUH, and followed the guidelines of the Declaration of Helsinki Principles.

\section{Results}

A total of 67 patients (age range, 3-82 years) had symptoms and signs suggestive of chlamydial conjunctivitis. Among the 67 patients, 45 (67.2\%, 95\% CI, 55.9-78.4) had positive DFA test results. Of these 45 patients, 42 with positive results received treatment, and the other 3 patients went abroad and did not receive treatment. The baseline patient data are shown in Table 1. After the first 2 weeks of treatment with oral azithromycin, only $27(64.3 \%, 95 \%$ CI, 49.8-78.8) of the 42 patients returned to the clinic and completed the treatment course. We recalled the 15 patients who were lost to follow-up after the first 2 weeks of treatment, and 9 patients claimed that they did not return to the clinic because their eye symptoms improved significantly. The remaining six patients could not be reached. After the first 2 weeks of oral azithromycin treatment, the DFA tests of $19(70.4 \%, 95 \% \mathrm{CI}, 53.2-87.6)$ of the 27 patients were negative, and the other $8(29.6 \%, 95 \% \mathrm{CI}, 12.4-46.9)$ patients whose DFA tests remained positive received additional augmented treatment before the results of the DFA tests became negative (Table 2). Among these eight

Table 2 Oral azithromycin treatment efficacy

\begin{tabular}{lcc}
\hline $\begin{array}{l}\text { Bacteriological efficacy (number of treatments } \\
\text { required) }\end{array}$ & $\begin{array}{c}\text { No. of } \\
\text { patients }\end{array}$ & $\%$ \\
\hline $\begin{array}{l}\text { Two weekly doses of azithromycin (2) } \\
\text { Augmented doses of azithromycin }\end{array}$ & 19 & 70.4 \\
$\quad \begin{array}{l}\text { One augmented dose (3) } \\
\text { Two augmented doses (4) }\end{array}$ & 4 & 14.8 \\
Total efficacy & 4 & 14.8 \\
\hline
\end{tabular}


patients with persistently positive DFA results, four required a single augmented treatment and the other four required two augmented treatments to achieve negative DFA results. Of the 45 patients with positive DFA test results, 3 (82, 76, and 63 years, respectively) had superior tarsal conjunctival scarring, corneal opacity, and pannus formation, which were consistent with the presentation of advanced trachoma. All three patients completed the oral azithromycin treatment; two of the three patients required a single augmented treatment, whereas the third patient (82 years old) required two augmented treatments. All the remaining patients (including the patients who were lost to follow-up) had translucent follicles on both superior and inferior tarsal conjunctiva without obvious conjunctival scarring or corneal pannus formation. Because $C$. trachomatis serotypes were not routinely examined in our central laboratory, it was difficult to differentiate trachoma from adult inclusion conjunctivitis by clinical presentation in these patients, except in three patients with advanced trachoma. In our study, we also analyzed the relationship between age and treatment effect of oral azithromycin. Older patients had a tendency to necessitate more augmented treatments (Figure 1). The oral azithromycin treatment was well tolerated by all patients. Only one patient had an episode of transient mild gastritis, and no other severe adverse events were observed.

\section{Conclusion}

C. trachomatis is one of the most commonly found infectious agents causing chronic conjunctivitis, and it can be subdivided into 15 serovars. Though different groups of serovars show unique tissue tropisms, they are not tissue selective. ${ }^{19}$ Clinically, it is difficult to diagnose the initial stages of trachoma or inclusion body conjunctivitis except by laboratory testing and confirmation. ${ }^{19}$ Because serotyping is not routinely

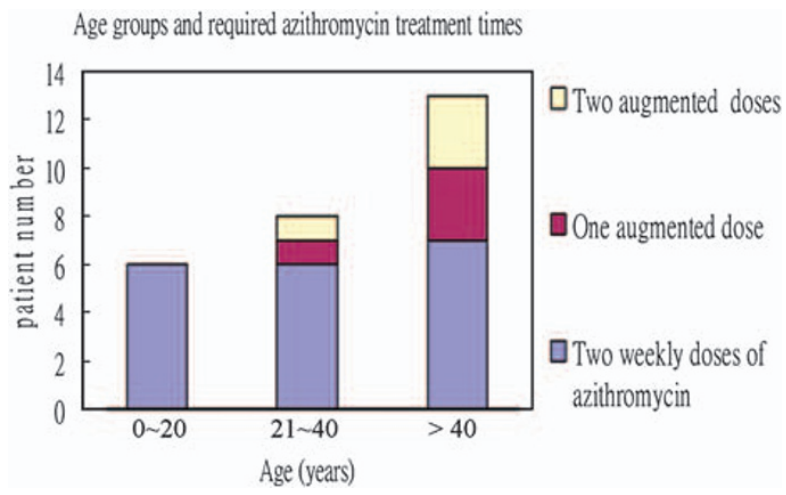

Figure 1 Relationship between age groups and required duration of oral azithromycin treatment. performed in our hospital, we were not able to differentiate the serovars of $C$. trachomatis in our patients. In our study, we used the DFA test because it is a rapid, sensitive, and simple method for the diagnosis of Chlamydia infection. ${ }^{20}$ Though the DFA test might be a little less specific with more false-positive results compared with C. trachomatis culture for test-of-cure analysis, DFA may be useful in the initial testing of patients after antimicrobial therapy. ${ }^{21}$ In a previous study by Schachter et al ${ }^{8}$, a marked decrease in C. trachomatis infection in endemic areas was achieved by the use of oral azithromycin once a week for 3 weeks. In our outpatient clinic, we initially attempted to treat our patients with oral azithromycin once a week for 2 weeks, after the diagnosis was confirmed positive by the DFA results. The review of the medical records revealed that the bacteriological eradication rate was high after the first 2-week course of oral azithromycin treatment $(70.4 \%$, 95\% CI, 53.2-87.6), but not as high as reported elsewhere. ${ }^{11,15}$ For example, Katusic et al ${ }^{11}$ reported a C. trachomatis eradication rate as high as $92 \%$ with only a single dose of oral azithromycin. They evaluated the eradication rate of $C$. trachomatis 10-12 days after the initial treatment, which was 2-3 weeks earlier than in our study. Thus, the possibility of re-infection and relapse of the disease may account for different eradication rates in our patients. Moreover, patients with persistent infection and clinical symptoms tended to return for follow-up and further treatment. In our study, we had a high rate of patients who were lost to follow-up $(35.7 \%, 95 \%$ CI, 21.2-50.2) after the first treatment. This may also explain the relatively low rate of eradication of Chlamydia after the first treatment in our patients. In this study, we attempted to recall the 15 patients who were lost to follow-up after the first 2 weeks of treatment and were thus excluded from our study to check their final DFA results before the study data analysis. As none of them returned for examination, we were unable to know the final DFA status of these excluded patients.

Furthermore, because most of these patients were lost to follow-up for more than 6 months, some potential bias associated with this lag, including the disease relapse, re-infection, or lack of adequate augmented treatments, might also influence the final DFA results of these patients. As these 15 patients were lost to followup after the first oral azithromycin treatment in our study, a worst-case scenario sensitivity analysis was performed assuming that all 15 patients still had positive DFA results after the first treatment. This resulted in a treatment efficacy of $45.2 \%$ (95\% CI, 30.2-60.3) after the first 2 weeks of oral azithromycin. In contrast, if the DFA results for all 15 patients had been negative after the first oral azithromycin treatment, the best-case scenario sensitivity analysis would have 
resulted in a treatment efficacy of $81.0 \%$ (95\% CI, 69.1-92.8).

In our study, approximately $30 \%$ of the patients still had positive DFA results even after the 2-week course of weekly oral azithromycin treatment, suggesting that these patients required augmented treatments.

Augmented oral azithromycin treatments were necessary in some patients for several reasons. First, patients' poor compliance might be taken into consideration, but it seems unlikely as these patients were administered oral azithromycin once a week only. Second, the possibility of relapse or re-infection in these patients could not be completely ruled out. As C. trachomatis is contact transmitted, any infected family member might have been the source of re-infection if they were not treated. In our opinion, all patients should be advised to inform their family members to undergo diagnostics and treatment for possible chlamydial infection. Third, in the treatment of trachoma worldwide, researches had found that rounds of mass treatment with single-dose azithromycin in trachoma-hyperendemic areas cannot eliminate trachoma or ocular $C$. trachomatis but can lower the incidence of infection in the long term. ${ }^{22-24}$ In our study, there might also have been some patients with very high loads of $C$. trachomatis for whom a 2-week course of weekly azithromycin might have been insufficient to eradicate the infection. For these patients, augmented azithromycin may help eliminate the infection. Fourth, in this study, we used the Chlamydia (genus)-specific reagent (bioMérieux) for the DFA tests rather than the $C$. trachomatis-specific major outer membrane protein reagent for the detection of chlamydial infection in the central laboratory of our hospital. It means that trachomatis and non-trachomatis Chlamydia (ie, C. pneumoniae and C. psittaci) are difficult to be distinguished by our DFA tests. It is possible that some of our patients who needed augmented treatments might be due to non-trachomatis Chlamydia infection, as non-trachomatis chlamydial conjunctivitis is thought more common than previously understood, and a longer course of antibiotic treatment than $C$. trachomatis infection was considered necessary to eradicate the organism. $^{25,26}$

In this study, we also observed that older patients had a tendency to require more augmented treatments, but the number of patients was too small to draw a definite conclusion. Further well-powered clinical studies with a larger number of participants are required to confirm this association.

The results of this study suggest that in the treatment of chlamydial conjunctivitis oral azithromycin treatment is effective and well tolerated. However, augmented oral azithromycin treatment may be necessary in some patients before chlamydial conjunctivitis can be treated.

\section{Summary}

\section{What was known before}

- The use of topical tetracycline ointments or oral tetracycline/doxycycline/erythromycin is the treatment for trachoma but the compliance may be poor.

- The oral azithromycin is effective in the treatment of both trachoma and adult inclusion conjunctivitis.

\section{What this study adds}

- Two weekly doses of oral azithromycin were effective and well tolerated in the treatment of chlamydial conjunctivitis.

- Older patients had a tendency to require more augmented treatments in our study.

\section{Conflict of interest}

The authors declare no conflict of interest.

\section{References}

1 Mabey DC, Solomon AW, Foster A. Trachoma. Lancet 2003; 362: 223-229.

2 Resnikoff S, Pascolini D, Etya'ale D, Kocur I, Pararajasegaram R, Pokharel GP et al. Global data on visual impairment in the year 2002. Bull World Health Organ 2004; 82: 844-851.

3 MacCallan AF. Inclusion conjunctivitis. Br J Ophthalmol 1942; 26: 271-275.

4 Rao SK, Madhavan HN, Padmanabhan P, Lakshmi GS, Natarajan K, Garg D. Ocular chlamydial infections: clinicomicrobiological correlation. Cornea 1996; 15: 62-65.

5 Taylor HR. Trachoma - the future for a disease of the past. Br J Ophthalmol 1993; 77: 66-67.

6 Bailey RL, Arullendran P, Whittle HC, Mabey DC. Randomised controlled trial of single-dose azithromycin in treatment of trachoma. Lancet 1993; 342: 453-456.

7 Tabbara KF, Abu-el-Asrar A, al-Omar O, Choudhury AH, al-Faisal Z. Single-dose azithromycin in the treatment of trachoma: a randomized, controlled study. Ophthalmology 1996; 103: 842-846.

8 Schachter J, West SK, Mabey D, Dawson CR, Bobo L, Bailey $\mathrm{R}$ et al. Azithromycin in control of trachoma. Lancet 1999; 354: 630-635.

9 Solomon AW, Holland MJ, Alexander ND, Massae PA, Aguirre A, Natividad-Sancho A et al. Mass treatment with single-dose azithromycin for trachoma. N Engl J Med 2004; 351: 1962-1971.

10 World Health Organization. World Health Assembly Resolution WHA 51.11. World Health Organization: Geneva, 1998.

11 Katusic D, Petricek I, Mandic Z, Petric I, Salopek-Rabatic J, Kruzic $V$ et al. Azithromycin vs doxycycline in the treatment of inclusion conjunctivitis. Am J Ophthalmol 2003; 135: 447-451.

12 Nakagawa H. Treatment of chlamydial conjunctivitis. Ophthalmologica 1997; 211: 25-28.

13 Peters DH, Heather AF, McTavish D. Azithromycin: a review of its antimicrobial activity, pharmacokinetic properties and clinical efficacy. Drugs 1992; 44: 750-799.

14 Hammerschlag MR, Gelling M, Roblin PM, Kutlin A, Jule JE. Treatment of neonatal chlamydial conjunctivitis with azithromycin. Pediatr Infect Dis J 1998; 17: 1049-1050. 
15 Salopek-Rabatic J. Chlamydial conjunctivitis in contact lens wearers: successful treatment with single dose azithromycin. CLAO J 2001; 27: 209-211.

16 Assaad FA, Maxwell-Lyons F, Sundaresan T. Report of 4 years' follow-up of a trachoma clinical trial in Taiwan. Bull World Health Organ 1968; 38: 565-576.

17 Woolridge RL, Wang SP, Grayston JT. Trachoma virus isolation studies on Taiwan. Bull World Health Organ 1962; 26: 783-787.

18 Wang HZ, Tsai RK, Jeng JE, Sheu MM, Huang WL, Lin CP et al. The re-evaluation of the prevalence of trachoma in primary school children in Kaohsiung City. Kaohsiung J Med Sci 1995; 11: 322-329.

19 Zinkernagel M, Catalano E, Ammann-Rauch D. Late-stage inclusion body conjunctivitis: trachoma? Br J Ophthalmol 2007; 91: 402-403.

20 Tam MR, Stamm WE, Handsfield HH, Stephens R, Kuo CC, Holmes KK et al. Culture-independent diagnosis of Chlamydia trachomatis using monoclonal antibiotics. N Engl J Med 1984; 310: 1446-1450.

21 Nachamkin I, Sawyer K, Skalina D, Crooks GW, Ciotti R, Sondheimer SJ. Test-of-cure analysis by direct immuno- fluorescence for Chlamydia trachomatis after antimicrobial therapy. J Clin Microbiol 1987; 25: 1774-1775.

22 Burton MJ, Holland MJ, Makalo P, Aryee EA, Alexander ND, Sillah A et al. Re-emergence of Chlamydia trachomatis infection after mass antibiotic treatment of a trachoma endemic Gambian community. Lancet 2005; 365: 1321-1328.

23 West ES, Munoz B, Mkocha H, Holland MJ, Aguirre A, Solomon AW et al. Mass treatment and the effect on the load of Chlamydia trachomatis infection in a trachoma-hyperendemic community. Invest Ophthalmol Vis Sci 2005; 46: 83-87.

24 West SK, Munoz B, Mkocha H, Gaydos C, Quinn T. Trachoma and ocular Chlamydia trachomatis were not eliminated three years after two rounds of mass treatment in a trachoma hyperendemic village. Invest Ophthalmol Vis Sci 2007; 48: 1492-1497.

25 Lietman T, Brooks D, Moncada J, Schachter J, Dawson C, Dean D. Chronic follicular conjunctivitis associated with Chlamydia psittaci or Chlamydia pneumoniae. Clin Infect Dis 1998; 26: 1335-1340.

26 Dean D, Kandel RP, Adhikari HK, Hessel T. Multiple Chlamydiaceae species in trachoma: implications for disease pathogenesis and control. PLoS Med 2008; 5: e14. 